\title{
VISUALISATION, SUBJECTIVITÉ ET MALADIE
}

\author{
Ch. Joussellin \\ Université Paris-Diderot, France \\ charles.joussellin@aphp.fr \\ F. Mailenova \\ Institut de Philosophie, Académie russe des Sciences, Russie \\ farida.mailenova@mail.ru \\ O. Popova \\ Institut de Philosophie, Académie russe des Sciences, Russie \\ j-9101980@yandex.ru
}

La santé et la maladie sont des facteurs subjectifs qui se développent au plus profond du sujet, et ce n'est qu'alors qu'ils sont exprimés à l'extérieur. La ligne de démarcation entre normal et pathologique est inexacte pour un groupe de personnes : où s'arrête la santé ? Ceci peut être évalué à la fois objectivement, par la recherche et le diagnostic, et en fonction du sentiment intérieur du sujet.

Il est également extrêmement important de tenir compte de ce que la personne ressent et dit pendant le traitement. Un langage imagé aide à entrer en contact avec le corps. Les capacités créatives d'une personne peuvent non seulement rendre sa vie flus brillante et plus heureuse, mais aussi l'aider à surmonter la maladie. Les psycho-technologies telles que les métaphores thérapeutiques sont un moyen étonnant d'utiliser l'imagerie pour se g’érir et se guérir soi-même.

Mots-clés: bioéthique, maladie, santé, subjectivité, autonomie, psychotechnologie, visualisation, métaphores thérapeutiques, communication humainistique, santé. 


\title{
ВИЗУАЯИЗАЦИЯ, СУБЪЕКТИВНОСТЬ И БОЯЕЗНЬ
}

\author{
Ш. Жюслен \\ Университет Париж-Дидро, Франция \\ charles.joussellin@aphp.fr \\ Ф. Майденова \\ Институт философии Российской академии наук, Россия \\ farida.mailenova@mail.ru \\ О. Попова \\ Институт философии Российской академии наук, Россия \\ j-9101980@yandex.ru
}

В статье предлагается взгляд на болезнь и здоровье как на субъективные факторы, имеющие к тому же своё образное выражение, которые развиваются глубоко внутри субъекта и лишь впоследствии получают своё выражение вовне. Размышляя о границе между нормальным и патологическим, которая всегда неточная и приблизительна, авторы задаются вопросом: где заканчивается здоровье? Это можно оценить как объективно, с помощью исследований и диагностики, так и согласно внутреннему ощущению самого субъекта. Хотя современная медицина и старается все более точно измерить и формализовагь процесс постановки диагноза, практикующие врачи вынуждены признать, что субъективный фактор вдияет как на изначальное восприятие болезни (и здоровья), так и на последующее чзлечение. При лечении также чрезвычайно важно учитывать то, что человек чувствует и говорит, каков ход его мыслей и в целом характер его убеждений. Для того чтобы понимать процессы, происходящие в теле, лучше всего подходит образный язык, который помогает получить контакт с телом. Отдельный раздел посвящён проблемам биоэтики, в котором подчеркивается, что в современном мире понимание болезни и особый ее статус определяются особым отношением к пациенту как к автономному существу. В биомедицинской этике принцип автономии приобретает особое значение, которое артикулируется на фоне традиционного медицинского патернализма. Уважение автономии дичности больного является стержневым ее понятием. Патернализм лишал больного права на обладание собственным телом и, по сути, отдавал его в распоряжение врача. Наблюдалась интересная ситуация: обладая правом на неприкосновенность к собственному телу, распоряжаясь им как собственностью в обычной жизни, человек терял это право, испытывая воздействие раздичных медицинских манипуляций. Сделан вывод о том, что в пространстве современной биомедицины возникает потребность возрастной дифференциации правовых и моральных норм как норм, имеющих контакт с раздичными реалиями телесного, с телесностью, обладающей раздичной семантикой. Фундаментальным оказывается тот факт, что морадь по-разному применима к человеческим 
существам, которые обладают раздичными телесными (биологическими) характеристиками. При этом информированное согласие касается только тех человеческих существ, которые подпадают под категорию автономных. При этом проблема манипуляций над человеческим телом не снимается полностью: фактически телесность независима от очевидных манипуляций, пока существует некая конвенциональная договоренность (дюдей экспертов - «во имя знания») относительно обладания полнотой автономии полноценности, нормализации по определенным критериям (возрастным, психическим, физическим параметрам). Дополнительное внимание уделено психологическим аспектам исследуемой проблемы, в частности влиянию убеждений на самочувствие больного. Исследование психодогического фактора подчеркивает важнейший тезис данной статьи о главенствующей роли субъекта. Терапевтические метафоры, используемые в лечении, являются действенными благодаря тому, что в этой технике исподьзуются возможности творческого бессознательного. Творческие возможности человека способны не только сделать его жизнь ярче и счастливее, но и помочь преодолеть болезнь. Психотехнологии, такие как работа с терапевтическими метафорами, являются тем удивительным способом использования образного мышления с целью исцеления и самоисцеления. Развитие телесного интеллекта очень важно не только в случае болезни или ее профилактики, но и в целом для улучшения качества жизни.

Кдючевые слова: биоэтика, болезнь, здоровье, субъективность, автономия, психотехнологии, визуализация, терапевтические метафоры, гуманистическое общение, здоровье.

\title{
VISUALIZATION, SUBJECTIVITY AND DISEASE
}

\author{
Charles Joussellin \\ Paris Diderot University, Paris, France \\ charles.joussellin@aphp.fr
}

Farida Mailenova

Institute of Philosophy of the Russian Academy of Sciences,

Moscow, Russian Federation

arida.mailenova@mail.ru

\section{Olga Popova}

Institute of Philosophy of the Russian Academy of Sciences,

Moscow, Russian Federation

j-9101980@yandex.ru

The article offers a view on the disease and health as subjective factors with their own imagery, which develop deep inside the subject and only then get their 
expression outside. Thinking about the boundary between the normal and the pathological, which is always inaccurate and approximate, the authors ask the question: where does health end? This can be assessed both objectively, through research and diagnosis, and according to the subject's own inner feeling. Although modern medicine is trying to measure and formalize the process of diagnosis more and more accurately, practitioners have to admit that the subjective factor influences both the initial perception of the disease (and health) and the subsequent healing. It is also extremely important to take into account what a person feels and says about their thoughts and the nature of their beliefs in general. In order to understand the processes taking place in the body, an imaginative language that helps to get in contact with the body suits best. A separate section is devoted to bioethical issues; it underlines that, in the modern world, the understanding of the disease and its special status is determined by the special attitude to the patient as to an independent being. In biomedical ethics, the principle of autonomy acquires special significance, which is articulated against the background of traditional medical paternalism. Respect for patient autonomy is a core concept of patient autonomy. The article concludes that, in the space of modern biomedicine, there is a need for age differentiation of legal and moral norms as norms having contact with different realities of the corporal, with the body, possessing different semantics. Fundamental to this is the fact that morality applies differently to human beings who have different bodily (biological) characteristics. It is emphasized that informed consent applies only to human beings who fall into the category of autonomous beings. At the same time, the problem of manipulation of the human body is not completely eliminated: in fact, the body matter is independent of obvious manipulations as long as there is some conventional agreement (of experts, "in the rame of knowledge") regarding the possession of full autonomy: fullness, normalization according to certain criteria (age, mental, physical and visual parameters). Additional attention in the article is paid to the psychological aspects of the problem under study, in particular, the impact of beliefs on the patient's health. The study of the psychological factor emphasizes the most important thesis of this article about the dominant role of the subject. Therapeutic metaphors used in treatment are effective due to the fact that this technique uses the capabilities of the creative unconscious. A person's creative abilities can not only make their life brighter and happier, but also help to overcome the disease. Psycho-technologies, such as working with therapeutic metaphors, are an amazing way to use imaginative thinking for healing and self-healing. The development of body intelligence is very important not only in the case of illness or prevention, but also in general to improve the quality of life.

Keywords: bioethics, disease, health, subjectivity, autonomy, psychotechnology, visualization, therapeutic metaphors, humanistic communication, health.

DOI 10.23951/2312-7899-2019-4-156-171 


\section{Introduction}

Sans nier les modifications physiologiques rencontrées, la santé et la maladie sont des phénomènes vivants évoluant au cœur de la subjectivité de chaque homme. Comment approcher la subjectivité d'autrui alors que celle-ci s'exprime déjà par la maladie, par son langage, essence de l'homme, et son activité artistique [Arendt 1983, 90], lesquels peuvent se déployer au cours d'exercice mentaux de visualisation et ainsi ouvrir un champ de possibles changements bénéfiques.

Abordons ce que représentent la santé, la maladie et la subjectivité, mais aussi ce qui relie la visualisation à l'activité artistique, pour proposer un cheminement à visée thérapeutique au cœur de la subjectivité.

Les capacités créatives d'une personne peuvent non seulement rendre sa vie plus lumineuse et plus heureuse, mais aussi l'aider à surmonter la maladie. Travailler avec des métaphores thérapeutiques est la façon étonnante d'utiliser la pensée de l'imagination pour la guérison et l'auto-guérison.

\section{La santé}

L'homme habite un monde vivant qu'il constitue aussi en existant et coexistant par ses relations et interrelations avec les autres êtres vivants, dont les autres hommes. Suivant les thèses de Georges Canguilhem, la maladie d'un homme n'est pas une variation sur une échelle normée de la santé, elle est une nouvelle dimension de la vie, une nouvelle allure de vie. " La maladie est ébranlement et mise en péril de l'existence » [Canguilhem 2010, 121] et seule la personne malade peut distinguer où commence la maladie. La frontière entre le normal et le pathologique est imprécise pour un groupe de personnes : où finit la santé ? où commence la maladie ? Limites dynamiques et polémiques parce qu'elles sont vivantes et subjectives. Phénomène vivant, la maladie n'a pas de sens si la conscience humaine, de la personne malade, des observateurs et des soignants, est mise de côté. En revanche, pour une seule personne à la fois, la frontière est précise et elle-même en est juge [Canguilhem 2010, 119]. C'est pourquoi en matière de santé, ce qui se montre doit être analysé à la lumière de ce qu'en dit la personne.

C'est particulièrement évident dans le counseling psychologique. Avant d'entrer dans le vif du sujet, il est toujours utile de savoir quelle est la norme pour le patient, c'est-à-dire quel serait le résultat de 
l'intervention du psychologue. De cette façon, nous obtenons deux points principaux pour le travail : le soi-disant état réel dans lequel le client a un inconfort, un problème, une douleur et l'état désiré, dans lequel il n'y a ni douleur ni inconfort et le problème est résolu. Le thérapeute ne peut pas savoir avec certitude à quel point son client est malade ou douloureux, il est obligé de le croire sur parole et de faire une image de la maladie à partir des paroles du patient. De plus, même en matière de guérison, il vaut mieux pour lui de s'appuyer non seulement sur des connaissances scientifiques objectives sur les maladies, mais aussi sur des connaissances subjectives internes, qui sont dans le cœur de chaque individu. Le meilleur expert sur lui-même est l'individu. Cependant, cette connaissance est souvent non identifiée et inconsciente, et on trouve souvent dans le travail que l'image/contenu de l'état souhaité est beaucoup moins clair que l'image de la douleur.

Cependant, le rôle du thérapeute est aussi d'aider le patient à mieux se comprendre et de l'accompagner sur son chemin vers la connaissance et la guérison.

\section{Douleur, maladie et autonomie}

« La maladie objective un rapport conflictuel au social » [Adam, Herzlich 2010, 64]. Vivante et parfois rebelle lorsqu'elle résiste aux techniques médico-chirurgicales mises en œuvre, la maladie peut déranger l'institution, la science et ses techniques. Pourtant la médecine, médiation et médium, ne se réduit pas à des techniques aussi savantes soient-elles. La maladie, celle d'une personne, expression d'un déséquilibre de sa relation avec l'environnement, demandera au médecin d'écouter et d'entendre la personne: " "faire avec" tel symptôme présenté par tel patient; éclairer ce symptôme par le discours de ce patient et ne plus lire à travers la grille scientifique qui réduit chaque corps à un corps anonyme » [Raimbault 1982, 27]. Accueillir la mise en récit de l'éprouvé d'une maladie représente le premier geste soignant. La maladie, au cœur de la subjectivité de la personne, est toujours aussi une construction socio culturelle signifiante qu'il faut reconnaitre, au sens d'accueillir.

Dans le monde moderne, la conception de la maladie et de son statut particulier est déterminée par le regard particulier du patient en tant qu'être autonome.

En éthique biomédicale, le principe d'autonomie acquiert une signification particulière, qui s'articule dans le contexte du paternalisme médical 
traditionnel. Le respect de l'autonomie du patient est un concept fondamental de l'autonomie du patient. Le paternalisme a privé le patient de son droit de posséder son propre corps et, en fait, l'a mis à la disposition du médecin. Une situation intéressante a été observée : ayant droit à l'inviolabilité de son propre corps, le disposant comme bien dans la vie quotidienne, une personne a perdu ce droit, subissant l'impact de diverses manipulations médicales. Le principe du respect de l'autonomie du patient, qui est au cœur de la bioéthique et qui est en contradiction avec le paternalisme médical traditionnel d'Hippocrate, a donné la priorité à la protection des droits du patient, avec la subordination jusqu'alors incontestée à l'autorité du médecin qui l'excluait du processus décisionnel concernant sa santé. Dans le contexte de la pensée libérale, la bioéthique redonne au patient le droit de posséder son propre corps en tant qu'individu, ce qui est consacré par la loi et l'éthique dans l'obligation d'obtenir le consentement informé du patient à toute intervention médicale.

M. M. Bakhtin dans son ouvrage «À la philosophie de l'action » a noté que chaque norme de substantive devrait être spécifiquement justifiée dans sa valeur par les sciences correspondantes: logique, esthétique, biologie, médecine, une des sciences sociales. Dans le contexte de la bioéthique, une telle idée devient encore plus radicale : chaque norme substantive doit être spécifiquement justifiée dans sa signification par sa correspondance avec les caractéristiques du corps humain, ce qui représente la possibilité de l'application de la norme.

Le corps d'un enfant à venir, le corps d'un enfant, le corps d'un adulte, le corps d'une personne compétente et le corps d'un malade mental sont des réalités corporelles différentes qui exigent des normes différentes et souvent contradictoires. Chacun de ces patients a sa propre perception de la douleur et sa propre façon d'appréhender la maladie, et chacun d'eux doit avoir une approche spécifique pour faire face à la maladie, des normes spécifiques visant à établir une communication avec le patient, en tenant compte de son expérience personnelle.

Par conséquent, il est nécessaire de différencier les normes juridiques et morales selon l'âge, en tant que normes ayant un contact avec différentes réalités du corps, avec le corps, avec différentes sémantiques. Ce qui est fondamental, c'est que la morale s'applique différemment aux êtres humains qui ont des caractéristiques corporelles (biologiques) différentes. Le corps d'un embryon et le corps d'une personne capable comme deux types de corps très différents (physiologiquement et personnellement) suggèrent des critères moraux différents. C'est le 
second type qui donne lieu à une implication dans les relations morales et juridiques en tant que participant égal, partenaire, être autonome lié à la communication humaine universelle, alors que le premier est sous la garde paternaliste d'un adulte ou de diverses institutions qui compensent son certain degré d'insuffisance par rapport aux normes de compétence. A cet égard, dans les discussions libérales modernes sur le statut des embryons, les droits du fœtus-patient, l'euthanasie, l'image d'une personne et son identité sont doublés conformément aux idées de J. Locke - nous sommes capables d'identifier les sujets de moralité et ceux dont le moi moral est soit non articulé soit représenté chez le proche aidant (cela vaut pour les embryons, enfants, maladies graves, maladie mentale, etc).

En effet, il ne s'agit pas des droits de ces êtres eux-mêmes, mais du droit à la protection de ces êtres. En séparant l'identité d'une personne (en tant qu'identité d'une substance, $d^{\prime}$ 'une matière, dont se compose un corps humain concret) et l'identité d'une personne (définie par la présence de la conscience de soi), Locke semble donner la priorité dans le degré de plénitude ( $\mathrm{d}^{\prime}$ une personne au sens plein du terme) à la conscience de soi individuelle autonome, capable de se fixer des objectifs et d'entretenir avec les autres, laquelle, conformément au concept du droit naturel, est dotée des droits à la vie, la propriété et la liberté. L'utilisation des idées de Locke dans la pratique bioéthique peut donner lieu, par exemple, à des réflexions sur l'infériorité, l'infériorité et l'infériorité de ceux qui ne possèdent pas encore la conscience de soi, fusionnant avec leur propre corps (embryons) et ceux qui ne la possèdent plus pleinement (par exemple, les patients atteints de la maladie d'Alzheimer, ceux ayant un handicap mental, etc.).

L'inverse est vrai d'un être adulte autonome: à l'ère de la biotechnologie, il est caractéristique qu'un médecin doit équilibrer sa propre volonté envers la personne souffrante avec celle de la personne ellemême. Dans le contexte médical, la conscience s'est concentrée sur la position ponctuelle d'un "observateur ", pour qui le corps est placé à l'extérieur comme un « objet ». Ce corps ne peut être affecté par la conscience que par un autre corps extérieur, le medicament [Tichenko 2001, 177].

Nous soulignons que les origines de cette position comportementale et les connotations éthiques qui lui sont associées résident dans la « vision médicale » du corps comme mécanisme qui s'est formé à l'époque cartésienne et qui a ensuite été adopté par le matérialisme français. Cependant, en raison d'une telle attitude, le médecin lui-même ressemblait à certains égards à un mécanisme, car sa « techne » s'avérait être 
une éthique médicale d'entreprise donnée, elle ne correspondait pas aux préférences personnelles du patient et n'était pas déterminée par la position subjective du médecin traitant.

Marcel Enaff, assez critique du fonctionnement médicalement compris du corps, aborde un point très important de son discours sur l'histoire de la science médicale libertine de Sade : "Un libertin qui démembre le corps de sa victime fait ce qu'un chirurgien se considère en droit de faire au nom du savoir au nom de son désir. La seule différence est que la première reconnaissance scandaleuse de ce plaisir... Toute connaissance et toute technique qui choisit le corps comme objet n'émerge que si le plaisir même par lequel il naît et disparaît est dépassé » [Annaff 2005, 52-53].

La position radicale d'Annaff souligne l'impossibilité de tracer une ligne claire entre la violence pour la connaissance (et donc pas la violence au sens classique du terme) et la violence en tant que telle. La violence au nom de la connaissance médicale n'était pas liée à la sémantique de la violence, car le modèle paternaliste de la relation médecinpatient n'impliquait pas un autre (non violent, c'est-à-dire tenant compte de la volonté de la pratique du patient), elle était légitime et justifiée par le bien du patient. Un modèle dit de relation d'ingénierie entre le médecin et le patient a été établi, selon lequel le médecin, en raison de sa perception rationaliste, ne voyait pas le patient comme une personne à part entière, mais comme un mécanisme dont le fonctionnement était de restaurer sa «techné ». La connaissance bioéthique affirme que la règle du consentement éclairé est un certain degré d'indépendance du corps par rapport aux manipulations faites à l'aide des connaissances médicales. Cependant, nous insistons sur le fait que cette règle s'applique toujours aux sujets de moralité normalisés, ceux qui sont capables d'exprimer leur volonté en raison de la présence de la conscience de soi. Même pendant l'anesthésie, le sujet, en fait, cesse d'être normalisé et à part entière. Pour un chirurgien qui suit la logique $\mathrm{du}$ « au nom de la connaissance », un tel patient devient un " matériel d'apprentissage » ou un « champ chirurgical ». La science objective le corps de l'homme car c'est une condition de son observation [Joussellin, Mailenova 2018].

Ainsi, dans cet esprit de raisonnement, le consentement éclairé ne concerne que les êtres humains qui entrent dans la catégorie des êtres autonomes. En même temps, le problème de la manipulation du corps humain n'est pas complètement éliminé: en effet, l'intégrité corporelle est indépendante des manipulations évidentes, tant qu'il existe un arrangement conventionnel (de personnes d'experts - « au nom de la 
connaissance ») concernant la possession de la plénitude de l'autonomie - complétude, normalisation selon certains critères (âge, état mental, paramètres physiques).

L'autonomie moderne n'est pas seulement un concept de droit ou d'éthique. Il s'avère qu'il est esthétiquement coloré. L'accent commercial mis sur un corps esthétiquement normalisé, dont l'apparence est largement due à la technologie médicale, est une caractéristique de la culture consumériste moderne.

Comme le souligne Mike Fiterstone, «la culture de consommation se referme sur cette image, qui encourage les individus à adapter les stratégies instrumentales existantes pour lutter contre la décomposition et la décomposition (ce qui est également approuvé par la bureaucratie d'État, qui tente de réduire le coût des soins de santé en cultivant un culte du corps dans la société) et de combiner cela avec une vision du corps comme un moyen de plaisir et d'auto-expression » [Featherstone $1982,18]$.

En fait, l'homme moderne ne s'intéresse probablement pas tant à la possibilité de posséder son propre corps qu'à la possibilité d'être représenté en raison de cette possession non seulement de la nature factuelle de sa nature non régulée, mais aussi de donner au corps luimême un statut indépendant (bien que subordonné au commencement rationnel) - comme un objet esthétique qui se fixe un but, une performance qui inclut une variété potentielle d'images, qui place l'accent principal dans la sémantique de la culture.

Dans ce cas, l'autonomie de disposer de son corps s'avère être demandée par le sujet tant qu'il a la possibilité de représenter son essence dans sa signification esthétique. En l'absence de cette possibilité, il y a un changement dans la nécessité de disposer du corps dans son expression la plus intense : dans son abandon complet, son rejet par le droit de propriété (dans l'acte d'euthanasie).

\section{La communication humanistique comme principe et condition de la guérison}

La communication entre une personne et une personne est un phénomène courant et en même temps peut cacher de nombreux miracles et énigmes. C'est par la communication que l'on peut guérir une autre personne et la faire souffrir. La capacité de communication humanistique, basée sur un amour profond de l'individu et la croyance que chacun a le potentiel de se développer et de créer, est essentielle 
pour les médecins, les psychologues et tous ceux qui travaillent avec les patients.

La question légitime se pose de savoir comment de traiter les manifestations du mal, la haine envers soi-même et envers les autres. Selon E. Fromm [Fromm 1993, 167], l'homme ne devient méchante que si les conditions nécessaires à sa croissance et à son développement sont absentes. Le Mal n'est pas imminent, mais conditionné par le manque de bien du Bien, et apparaît comme le résultat 'fonction d'un échec dans la vie.

Pour le travail d'un psychothérapeute, cette disposition est d'une importance fondamentale. À la recherche profonde des manifestations négatives de la personne par rapport aux autres et par rapport à ellemême, il devient finalement clair que dans une racine de chaque acte il y a une intention positive, une aspiration dans l'amour, la compréhension, le bonheur. Ainsi, tout mouvement de l'âme, même destructeur, peut être « redirigé » vers une direction positive. Le principe de l'intention positive, appliqué en psychothérapie, suppose une prémisse aussi fondamentale, car même des sentiments destructeurs comme le ressentiment, le désir de vengeance, même le désir de mutiler ou de tuer quelqu'un, contiennent dans leur essence un désir insatiable d'attention, d'acceptation et d'amour.

Une autre prémisse importante de l'éthique humaniste est l'hypothèse qu'une personne a une motivation innée à grandir et à se développer, et n'a donc pas besoin de stimuli externes supplémentaires pour sa créativité. Cette capacité se manifeste et est utilisée en psychothérapie, en particulier lorsqu'on utilise des métaphores. Une fois en transe, le client est impliqué dans le processus créatif de création d'une métaphore thérapeutique qui décrit et transforme ensuite sa douleur. Non seulement le résultat final de la thérapie, mais même le processus de création lui-même est la guérison.

\section{L'art de la métaphore de la guérison}

La métaphore est souvent le langage le plus efficace pour communiquer avec les patients au sujet de cas compliqués où il n'y a pratiquement " pas assez de mots » pour expliquer et comprendre, ainsi que dans des situations qui sont si chargées émotionnellement que la conversation directe est impossible à cause de trop de sentiments.

Les métaphores de la thérapie permettent, d'une part, de s'abstraire des émotions associées à la douleur émotionnelle et physique et, d'autre 
part, d'impliquer une pensée rationnelle qui est parfois dépassée par trop de douleur.

Un psychothérapeute travaille avec les mots, en utilisant la capacité du mot non seulement pour refléter le monde intérieur de la personne, mais aussi pour le changer, pour aider à faire face à la douleur et guérir. Cependant, un mot mal compris ou mal compris peut conduire le thérapeute sur la mauvaise voie et non seulement ne pas résoudre le problème du patient, mais aussi causer un traumatisme supplémentaire.

Pour améliorer la qualité de la communication thérapeutique, il est important d'être capable d'utiliser correctement le langage, de poser des questions et d'écouter attentivement la réponse, car le langage a la capacité de coder des informations cachées dans l'espace inconscient à l'aide d'images.

La particularité de la communication psychothérapeutique est que le langage n'est pas seulement un moyen de communication, mais un outil qui permet d'accéder à l'inconscient, dans le domaine du « moi » humain, où l'information existe sous forme d'images, de sensations corporelles, et où les souvenirs de tous les événements passés sont stockés, les plus douloureux, traumatiques, les plus heureux et les plus stimulants.

La métaphore vous permet d'accéder et d'utiliser l'énergie de ces souvenirs, ainsi que de surmonter en douceur la résistance à la thérapie et de travailler sur les blessures. Grâce aux métaphores, il est possible d'accéder à des ressources personnelles cachées et de permettre au cerveau de recycler des problèmes complexes et de trouver une issue à des situations auparavant désespérées. Une autre valeur stratégique des métaphores est qu'elles permettent aux capacités créatives uniques de l'inconscient humain d'être vues et utilisées pour guérir. Même après avoir vécu la terrible et traumatisante expérience de la violence (émotionnelle, physique, sexuelle), qui est habituellement déplacée mais qui continue d'empoisonner la vie, l'individu est capable d'y retourner dans un espace métaphorique et de recycler cette expérience afin que la horrible situation passée cesse de jouer un rôle significatif dans la vie actuelle réelle.

Souvent, c'est la méthode de la métaphore qui permet de guérir des problèmes qui durent de nombreuses années. La souffrance à long terme est le résultat de quelque chose qui a empêché le cerveau de retravailler une expérience douloureuse au moment de la blessure. La raison, en principe, était le manque de ressources à ce moment-là: impossibilité physique ou mentale d'une autre réaction à ce moment-là (dans des situations de violence ou de catastrophe), le petit âge du patient, sa forte dépendance émotionnelle et/ou physique de la personne qui a été la cause 
du trauma. Cependant, à l'heure actuelle, bien que la menace appartienne au passé, les conséquences de ce traumatisme continuent de causer de la douleur et d'empêcher le patient de poursuivre sa vie normale, laissant derrière lui des souffrances passées qui sont « éternisées » par le corps sous la forme d'une douleur somatique.

Le fonctionnement de la métaphore est pour l'instant un mystère pour nous, peut-être qu'à l'avenir avec le développement des neurosciences nous pourrons révéler le voile de ce mystère, mais en attendant nous pouvons juger son efficacité directement par le résultat, l'amélioration de l'état du patient. Si l'expérience douloureuse a été redessinée, toute la situation de la vie change par magie lorsque la transe est terminée. La manière exacte dont les processus se déroulent dans l'espace inconscient ne peut être jugée qu'indirectement, car le client, bien qu'actif, peut parler et répondre aux questions et, dans une certaine mesure, observer lui-même, percevoir ce qui se passe à travers des images aux couleurs émotionnelles vives et reste dans le processus de la session entière dans un monde imaginaire spécial, semblable au monde des rêves ou de la méditation.

Bien que les techniques d'hypnose d'Erickson n'impliquent pas d'immersion en transe profonde, le travail principal qui se déroule dans l'espace intérieur du client disparaît souvent lorsque le processus est terminé. Cet oubli, c'est comme oublier des rêves qui peuvent être saturés d'émotions très vives, parfois lourdes et terribles, et comme si à la fin du spectacle, les rideaux s'enfonçaient progressivement, les sons se calment et le silence arrive... C'est peut-être là la sagesse particulière de notre nature, qui ferme notre conscience à la contemplation d'images trop lourdes de notre vie.

\section{La subjectivité}

La subjectivité représente la façon singulière avec laquelle la personne parle de sa maladie, mais aussi le vécu de la maladie elle-même. Une évaluation qualitative ou quantitative de la subjectivité est illusoire et toujours réductrice car objectivante : «[...] il n'est jamais possible d'expliquer si peu que ce soit la subjectivité, fût-elle réduite à du psychisme, c'est-à-dire à son ombre portée sur le monde, par du biologique » [Michel 1987, 230]. Après Aristote et Saint Augustin, le sujet est aujourd'hui perçu en premier selon un ordre naturel. Le pronom « Je » est ainsi pris en considération si nous voulons signaler à d'autres l'existence en nous de telle ou telle expérience : je pense, je vois, je souffre 
Autrement dit, nous relions la subjectivité à un phénomène qui procède de la vie, du vivant, de l'homme vivant parmi les autres hommes; toujours singulière à un moment donné, influencée par les enjeux, les circonstances et l'histoire de l'homme malade.

Alors que la maladie se déroule au cœur de la subjectivité et que la subjectivité s'exprime aussi à travers le langage et l'activité artistique de la personne, les liens entre la visualisation et l'activité artistique montreront l'intérêt d'une telle démarche pour soigner autrui.

\section{La visualisation : œuvre d'art}

La visualisation dont nous parlons est l'action de représenter mentalement des images visuelles qui ne sont pas présentes; acte possédant des caractéristiques communes à la création d'une œuvre d'art.

En nous appuyant sur la façon par laquelle Martin Heidegger décrit l'œuvre d'art [Heidegger 1962], nous retenons que si l'œuvre d'art est fondée sur l'apparence, sur une interprétation humaine, la visualisation l'est aussi. Si à l'évidence la visualisation n'est pas fabriquée de la main de l'homme, en revanche comme l'œuvre d'art elle «s'use » et «s'épuise » dans son " utilité », même si cet épuisement n'est pas de même nature puisque la visualisation est immatérielle.

La visualisation, comme l'œuvre d'art, s'installe et s'ouvre au monde, donne aux images formes et couleurs, familiarité et étrangeté, amplitude et petitesse. Les images font venir " l'afflux infatigué et inlassable » de ce qui se dévoile du monde et de la terre. L'ailleurs et le jadis sont plus forts que l'ici et le maintenant. L'espace s'ouvre alors que l'intimité se replie, les détails s'estompent, les couleurs changent, l'espace est sans limite. Le processus de création de ces images, comme de l'œuvre d'art, fait advenir un étant qui n'existait pas et qui n'adviendra plus par la suite. Une création originale, éphémère, radicalement subjective, non reproductible à l'identique, imprévisible qui permet d'ériger, de construire, d'ouvrir un champ des possibles. Un événement singulier riche de possibilités qui ne viennent pas du néant mais des déterminations retenues par la qualité de la disponibilité d'accueil et d'écoute de soi-même à cet instant, en lien éventuellement avec des suggestions d'autrui.

Sous la domination de l'image, du temps et de l'espace, du dehors et du dedans, mais aussi sous le signe de l'infini, la visualisation, similaire à une activité artistique, se déploie au cœur de la subjectivité et permet à la personne malade de se découvrir et de découvrir ce qui peut l'aider. 


\section{Conclusion}

Dans cet article, nous avons essayé de prêter attention à la composante communicative de la médecine moderne. L'espace personnel de la maladie et l'expérience personnelle de la douleur doivent correspondre à des pratiques communicatives spéciales visant à visualiser la souffrance, à générer des images et des métaphores visuelles. Des outils psychologiques de ce genre nous permettent de porter la maladie, de la jouer d'une manière artistique, de la travailler comme une œuvre d'art.

Il est important dans le processus d'interaction communicative entre un médecin et un patient de prendre en compte sa position existentielle autonome. Le patient n'est pas une construction d'ingénierie ou un instrument manipulable à l'infini. Un porteur de responsabilité et, en même temps, un artiste travaillant dans un espace communicatif avec des images du patient, avec le pouvoir de la métaphore et créant dans son image du monde un monde alternatif, plus harmonieux de douleur et de souffrance.

Le langage peut être précis, subtil et dangereux comme une arme. Ça peut faire mal comme un couteau et guérir comme un bistouri. Et les psycho-technologies existantes permettent de renforcer à plusieurs reprises ces propriétés de la langue, la conduisant parfois à la magie.

Le langage imaginatif, utilisé pour créer une transe dynamique en alternant l'accompagnement du client de l'espace de ses sensations corporelles à l'espace de la pensée créative avec le chargement de son régime alimentaire et son retour aux sensations du corps, permet de voir comment le langage peut devenir matériel et acquérir un pouvoir curatif. La connexion entre l'esprit et le corps par le langage est à la fois un miracle et un mystère, et ce qui nous arrive chaque jour. Mais en même temps, elle peut être explorée et reproduite, aidant les individus à apprendre et à guérir.

Grâce à la métaphore, nous pouvons faire un pas de plus dans la connaissance du merveilleux monde humain et ouvrir légèrement le voile sur les mystères étonnants de notre cerveau et de notre inconscient créatif. 


\section{LITTÉRATURE}

Adam, Herzlich 2010 - Adam P., Herzlich C. Sociologie de la maladie et de la medicine. Paris, Armand Colin, 2010.

Annaff 2005 - Annaff M. Marquis de Sade. L'invention du corps libertin. Saint-Pétersbourg : IC « Humanitarian Academy », 2005. (In Rus.)

Arendt 1983 - Arendt H. Condition de l'homme modern. Paris : CalmannLévy. 1983.

Canguilhem 2010 - Canguilhem G. Le normal et le pathologique. Paris : PUF. 2010.

Featherstone 1982 - Featherstone M. The Body in Consumer Culture // Theory, Culture and Society. 1982. Vol. 1 (2). P. 18-33.

Fromm 1993 - Fromm E. Un homme pour lui-même. Introduction à la psychologie de l'éthique. Livre : Psychanalyse et éthique. Moscou : «République », 1993. (In Rus.)

Heidegger 1962 - Heidegger M. L'origine de l'œuvre d'art. Chemins qui ne mènent nulle part. Paris, Gallimard, 1962. P. 13-98.

Joussellin, Mailenova 2018 - Joussellin Ch., Mailenova F. L'Objectivation du corps et nouvelles technologies // ПРА $\Xi H M A$. Journal of Visual Semiotics. 2018. № 4. P. 142-147.

Michel 1987-Michel H. La barbarie. Paris, Grasset, 1987.

Raimbault 1982 - Raimbault G. Clinique du réel, la psychanalyse et les frontières du médical, Paris, Seuil, 1982.

Tichenko 2001 - Tichenko P. D. Bio-pouvoir dans l'ère de la biotechnologie. Moscou, 2001. (In Rus.) 